\title{
Using Dimensionless Numbers to Predict Centrifugal Jet-Spun Nanofiber Morphology
}

\author{
Prashanth Ravishankar $(\mathbb{D}$, Alex Khang $(\mathbb{D}$, Melissa Laredo $(\mathbb{D}$, and Kartik Balachandran \\ Department of Biomedical Engineering, University of Arkansas, Fayetteville, AR 72701, USA \\ Correspondence should be addressed to Kartik Balachandran; kbalacha@uark.edu
}

Received 13 November 2018; Revised 1 February 2019; Accepted 25 February 2019; Published 22 April 2019

Academic Editor: Bo Tan

Copyright (C) 2019 Prashanth Ravishankar et al. This is an open access article distributed under the Creative Commons Attribution License, which permits unrestricted use, distribution, and reproduction in any medium, provided the original work is properly cited.

In this study, we report a method for predictive, controlled, and highly aligned nanofiber production via Centrifugal Jet Spinning (CJS) using polycaprolactone (PCL) as a model polymer. We investigated the effects of fabrication conditions and their resulting dimensionless parameters, namely the Weber, Reynolds, and Capillary numbers, by correlating with fiber morphologies (fiber diameter, fiber alignment, bead frequency, bead aspect ratio, and scaffold porosity) and mechanical properties (linear modulus and ultimate tensile strength). We report a fabrication parameter lookup table based on the aforementioned dimensionless numbers, for the production of nanofiber scaffolds using the CJS. We built a scaled-up version of the CJS that uses a larger reservoir and successfully validated the reported lookup table for PCL as well as other polymers including polyethylene oxide, polylactic acid, and polyvinylpyrrolidone dissolved either in hexafluoroisopropanol or chloroform. We show that by carefully tailoring the polymer intrinsic properties and the Reynolds number, we can fabricate bead-free, continuous fibers. This method will allow other researchers to design and build their own CJS for the production of desired fiber scaffold networks by utilizing the appropriate dimensionless numbers for their system.

\section{Introduction}

Nanofiber scaffolds are used in tissue engineering because of their high surface area to volume ratio, ability to integrate with the local tissue structures, tunable degradation, and mechanical properties $[1,2]$. Several techniques exist for the production of nanofibers like electrospinning, melt blowing, centrifugal jet spinning, phase separation, template synthesis, and nozzle-free rotary spinning [1-6]. Some of these techniques offer benefits that are more advantageous than the others. For instance, electrospinning is popular in the academic setting but involves high power consumption and low yield limiting the technique to be scaled up for industrial use [7]. Melt spinning requires the use of high temperatures and involves high power consumption [8]. Thus, a more efficient method that overcomes these limitations is needed. We focus on an alternative technique called centrifugal jet spinning (CJS), also known as rotary jet spinning, that has been shown to be more efficient because of its capability to reliably produce high quantities of nanofibers within a short duration at low cost and low power consumption and for its potential to be scaled-up to increase production rates [3, 5, 9-11]. CJS utilizes a high centrifugal force produced by a rotating reservoir to extrude nanofibers. By employing CJS, we have previously reported the fabrication of anisotropic, Janus-type polymer nanofiber scaffolds that featured distinct properties on either side [4]. One of the challenges in any nanofiber production process is understanding the role of the various fabrication parameters that determine the overall physical and chemical properties of the produced nanofibers. The principle behind the CJS process requires some basic knowledge of polymer chemistry, processing, and fluid mechanics [3, $8,12,13]$. Relatively few publications have studied the effect of these various parameters involved, and Figure 1 depicts the complex relationship between these parameters $[3,5,6$, 12-17].

To our knowledge, existing CJS literature has focused on studying the fibers collected on a distal collector, while our 


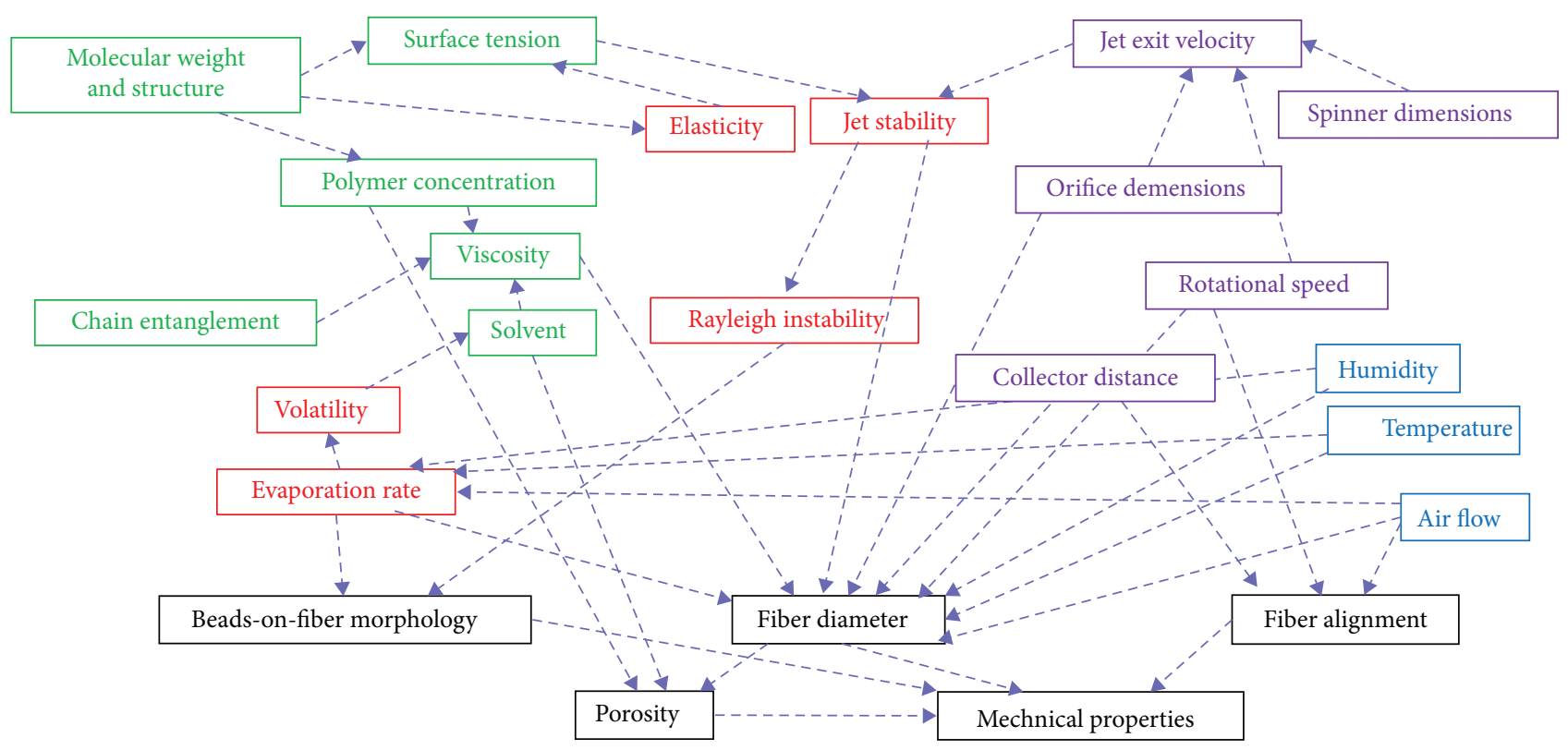

FIGURE 1: Schematic of the complex relationship between the ambient conditions (blue), polymer solution characteristics (green), process parameters (purple), and other underlying dependent variables (red) on scaffold characteristics (black) developed based on a review of published literature $[3,5,6,12-17]$.

study focuses on the highly aligned fibers that were collected on the rotating reservoir itself. We believe this paper will thus add more information on how the fiber fabrication parameters of these highly aligned nanofibers can be controlled in the CJS process.

In this study, we describe a method to utilize dimensionless parameters to reliably fabricate and scale-up nanofiber production via CJS. We hypothesized that by identifying and matching specific dimensionless numbers that characterize our system, we can predict the resulting nanofiber morphology. We chose polycaprolactone (PCL) as our initial model polymer as it is a widely studied polymer with diverse applications including wound dressings [17-19], anterior cruciate ligament reconstruction $[20,21]$, and tissue engineering of blood vessels [22]. We show that fiber morphology can be predicted by specific combinations of the intrinsic properties of the polymer solution like viscosity, density, and surface tension along with the CJS system operational conditions (rotational speeds, extrusion orifice diameter, and centrifugal forces) via the dimensionless Reynolds, Weber, and Capillary numbers. The Reynolds number (Re) represents the ratio of fluid inertial force to viscous force, and the Weber number (We) is the ratio of fluid inertial force to surface tension $[3,23]$. Here, the inertial forces are the forces due to the momentum of the fluid which is given by density and jet exit velocity of the polymer solution. The capillary number $(\mathrm{Ca})$ is the ratio of the Weber number over the Reynolds number and describes the role of viscous forces to surface tension forces $[3,8]$. These three dimensionless numbers are responsible for the thinning of the jet filaments ?exiting the rotating reservoir orifice and are proportional to the velocity that drives the formation of the polymer jet [24]. We use these dimensionless numbers to correlate with the fiber morphology and mechanical data to find the dimensionless number that has the most significant correlation. Using this significant dimensionless number, we generate a predictive lookup table to define the operating parameters for a larger scaled-up version of the CJS and validate using PCL and other polymer solutions made from polyethylene oxide, polyvinylpyrrolidone, and polylactic acid.

\section{Materials and Methods}

2.1. CJS Systems and Reservoir Nozzles. Two different CJS systems were used-a small-scale CJS prototype and a scaled-up CJS. The small-scale CJS (Figure 2(a)) spins a reservoir (Figure 2(b)) with an internal diameter of $26 \mathrm{~mm}$ and two orifices of equal diameter $(D=254 \mu \mathrm{m})$ at the bottom of the reservoir. This ?system used a commercial motor (Model EC22, Maxon Motor Ag, Switzerland) controlled by an Elvis II Workstation (National Instruments, USA) to control rotational speeds ranging from 10,000 to $25,000 \mathrm{rpm}$ yielding nanofibers (Figure 2(c)) via centrifugal extrusion and solvent evaporation represented in the schematic Figures 2(d) and 2(e) and outlined in detail in our previous publications [4, 25]. The scaled-up CJS prototype (Figure $2(\mathrm{f})$ ) was constructed with a commercial $300 \mathrm{~W}$ CNC Milling Spindle motor (KL-300, Automation Technologies Inc., USA) and controlled by an E300 series VFD (KL-VFD05, Automation Technologies Inc., USA) to vary the rotational speeds. The scaled-up system used a larger reservoir (Figure 2(g)) with an internal diameter of $48 \mathrm{~mm}$ and two orifices of equal diameter $(D=254 \mu \mathrm{m})$ on either side of the reservoir.

2.2. Polymer Solutions: Preparation, Analysis, and Calculations. All polymers and solvents were obtained from Sigma-Aldrich, Oakwood Chemical, and Alfa Aesar, unless 


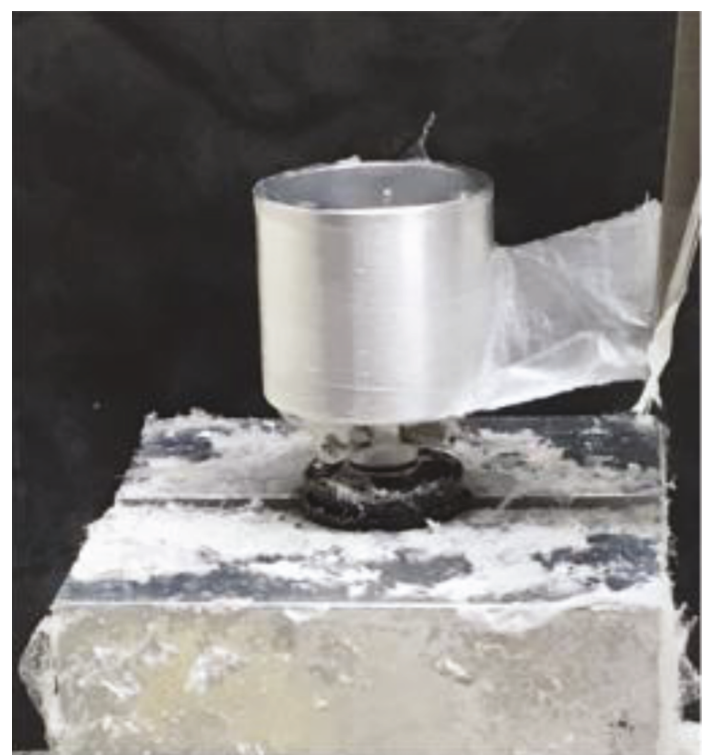

(a)

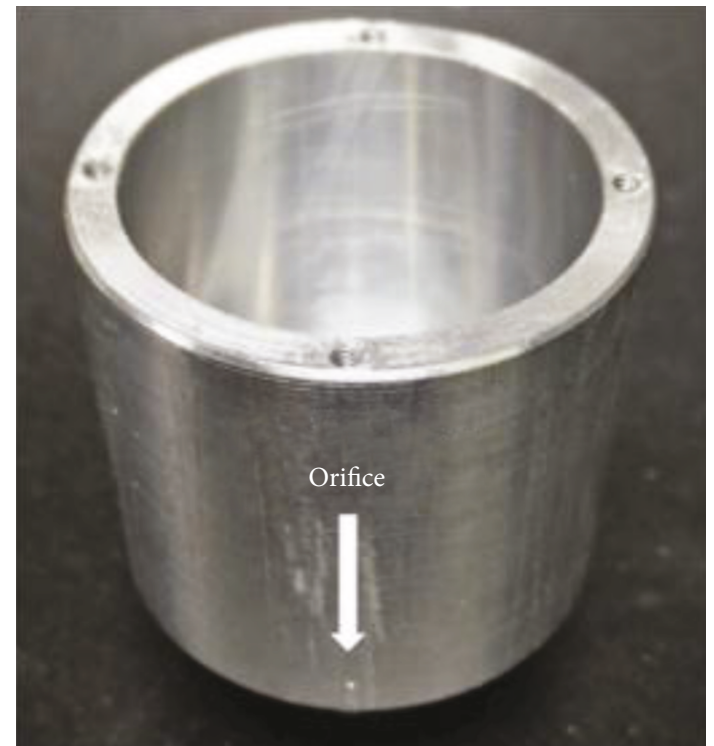

(b)

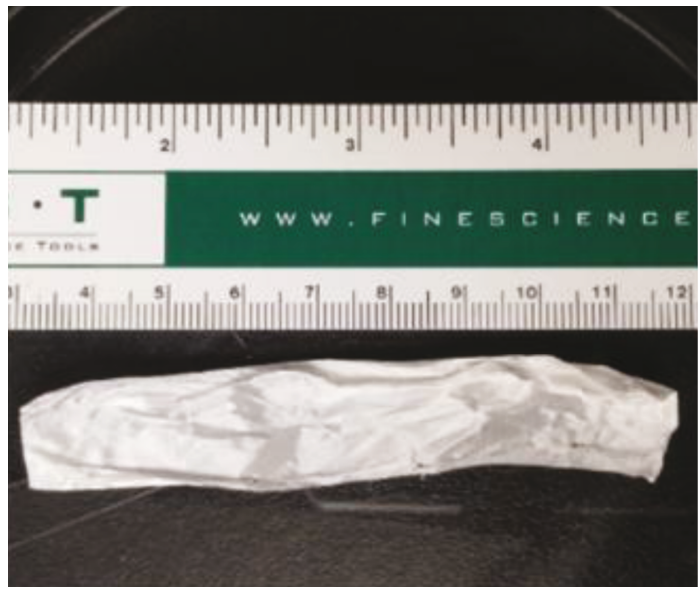

(c)

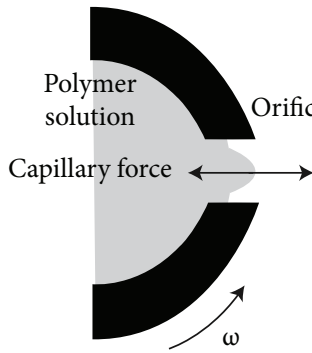

(i) Initiation

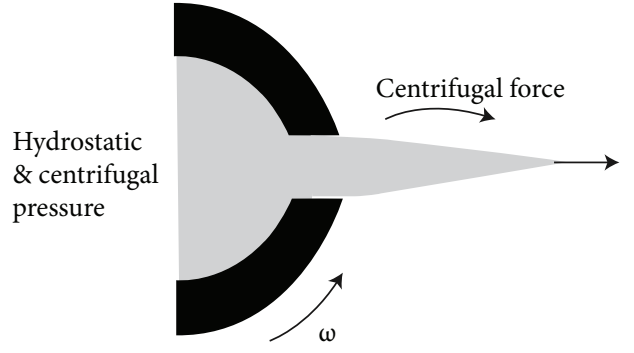

(ii) Extention

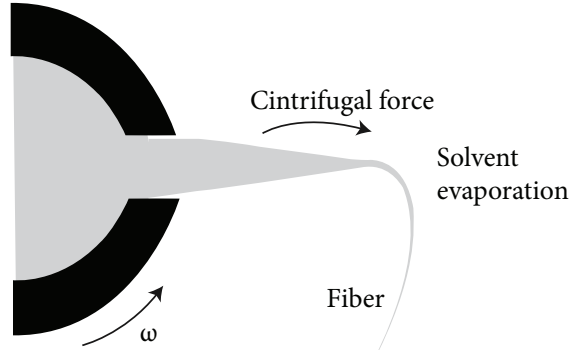

(iii) Evaporation

(d)

Figure 2: Continued. 


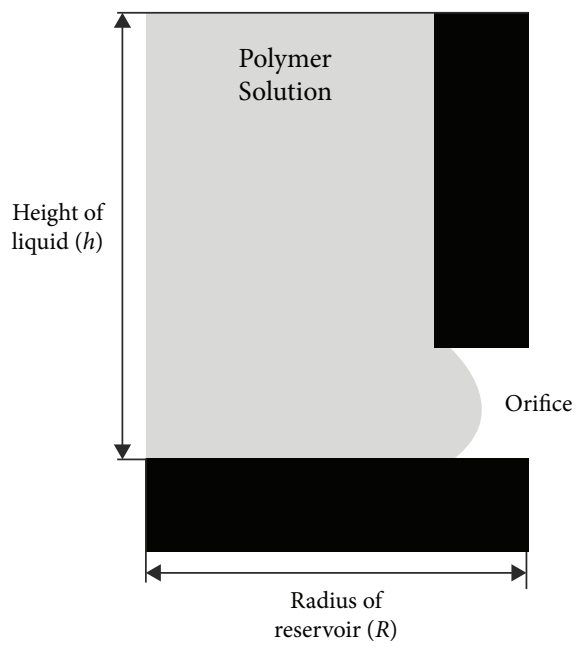

(e)

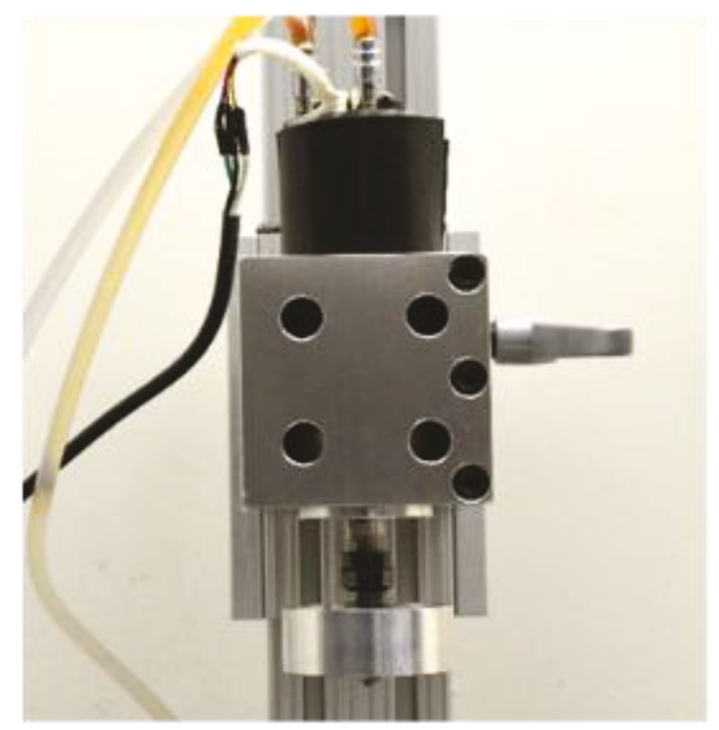

(f)

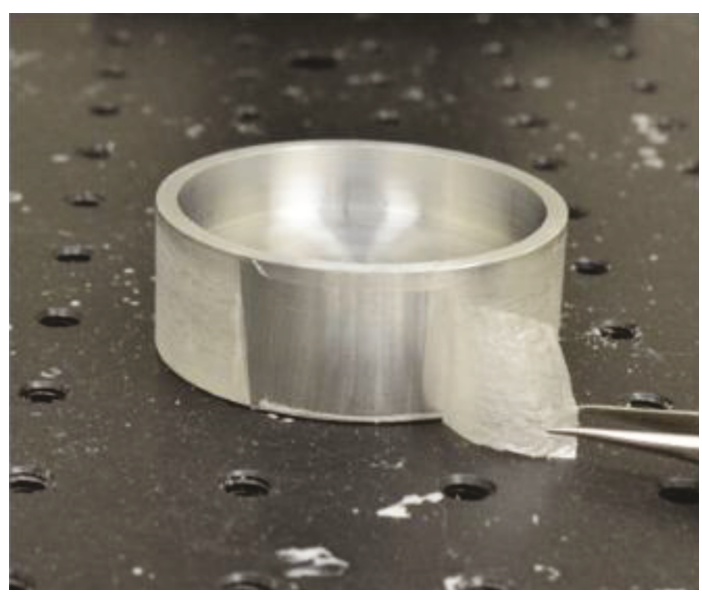

(g)

FIgure 2: (a) Small-scale CJS prototype used for fabricating nanofibers with the nanofibers being wound onto the reservoir eliminating the use of a collector. (b) The reservoir employed in the small-scale CJS with one of its orifice shown. (c) Representative image of a nanofiber mesh fabricated using the CJS prototype. (d) A top-down schematic of the reservoir depicting the mechanism of fiber formation. (e) Cross-sectional view illustrating the dimensions of the reservoir. (f) Scaled-up CJS system constructed for validation using a (g) larger reservoir. Similar to the CJS prototype, the fibers were wrapped around the scaled-up CJS reservoir.

otherwise specified. Polycaprolactone (PCL) was dissolved in 1,1,1,3,3,3-hexafluoro-2-propanol (HFIP) to make five distinct $4 \%, 5 \%, 6 \%, 7 \%$, and $8 \%(w / v)$ polymer solutions. Polymer solutions of polyethylene oxide- (PEO-) chloroform, polylactic acid- (PLA-) chloroform, low molecular weight (55,000 Da) polyvinylpyrrolidone- (PVP-L-) HFIP, and high molecular weight $(1,300,000 \mathrm{Da})$ polyvinylpyrrolidone(PVP-H-) HFIP were prepared at concentrations ranging from 3 to $10 \%(w / v)$. All solutions were magnetically stirred overnight to ensure the complete dissolution of the polymer. Polymer density $(\rho)$, dynamic viscosity $(\eta)$, and surface tension $(\gamma)$ of all polymer solutions were measured using previously published methods [4]. These measured values for the PCL model polymer are reported in Table 1.

2.3. Fabrication of Nanofibers Using Small-Scale CJS Prototype. The small-scale CJS reservoir was filled with
$10 \mathrm{ml}$ of polymer solution and operated for 150 seconds for each of the five PCL solutions at various speeds $(10,000$, 15,000, 20,000, and 25,000 RPM) under ambient temperatures to yield fibers. The fibers were collected from the reservoir surface (Figure 2(a)) and only areas unaffected by the tweezers were used for analysis [4].

\subsection{Scanning Electron Microscopy and Fiber Morphology} Characterization. Nanofiber sections were fixed onto a stud via carbon tape and sputter coated with gold. These coated samples were imaged at $15 \mathrm{kV}$ with a $5 \mathrm{~mm}$ working distance using a Nova Nanolab 200 (FEI, Hillsboro OR, USA) scanning electron microscope (SEM) to capture ten random image fields for each sample. The fiber orientation parameter (OP) and porosity were computed using custom-written MATLAB (MathWorks, USA) scripts to determine fiber alignment and overall scaffold porosity, respectively $[4,26]$. 


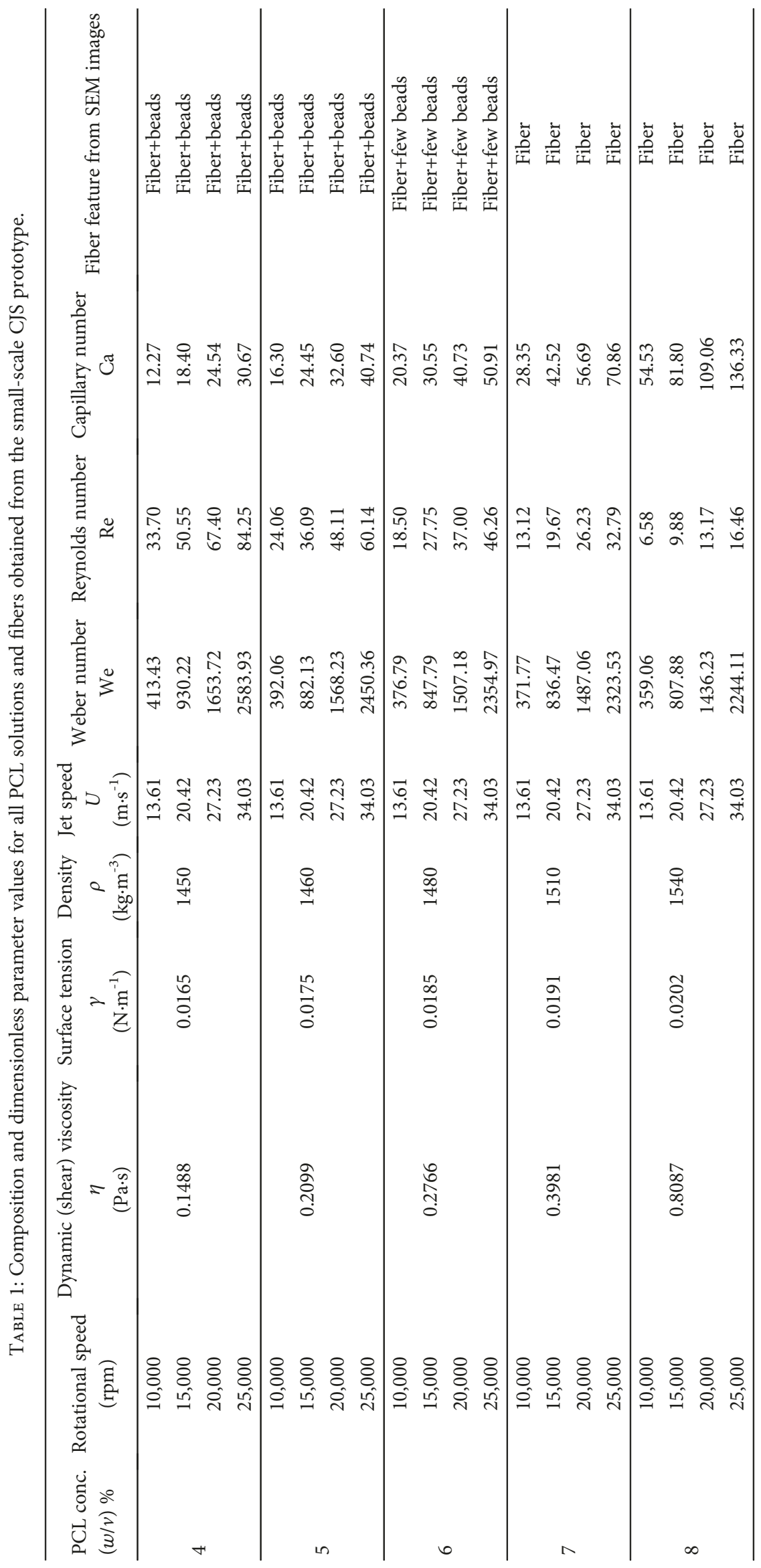


The SEM images were also analyzed for fiber diameter and beading using ImageJ [4]. Fiber diameter was analyzed by averaging 15 random fibers in each obtained image for each image field. For bead analysis, we calculated bead frequency by counting the number of beads normalized to the area (in $\mathrm{mm}^{2}$ ) in all nanofiber conditions and computed the bead aspect ratios using ImageJ.

2.5. Uniaxial Tensile Strength Testing. Rectangular nanofiber specimens $(20 \mathrm{~mm} \times 10 \mathrm{~mm} \times 0.05 \mathrm{~mm})$ were mounted on an Instron ${ }^{\circledR} 5900$ Series (Canton, MA, USA) tensile strength testing apparatus. Each sample was subjected to ten cycles of preconditioning to a minimum and maximum strain magnitude of 1 and 5\%, respectively, before being pulled at $10 \mathrm{~mm} / \mathrm{min}$ until failure. The first tear across the nanofiber construct marked the failure of the sample. The linear modulus (LM) was calculated from the slope of the resulting tensile stress vs. strain plots by choosing two points within the linear region of the curve, and ultimate tensile strength (UTS) was determined from the highest point on the curve [4].

2.6. Calculating Critical Polymer Chain Entanglement Concentration. To determine the critical concentration $\left(C_{\text {critical }}\right)$, which defines the concentration above which continuous bead-free nanofibers can be fabricated, the specific viscosity of polymers were plotted against polymer concentrations. Polymers were dissolved in their respective solvents at $3-10 \%(w / v)$, and their specific viscosities were measured. The specific viscosity was obtained by

$$
\eta_{\mathrm{sp}}=\frac{\left(\eta_{\text {Polymer solution }}-\eta_{\text {Solvent }}\right)}{\eta_{\text {Solvent }}}
$$

where $\quad \eta_{\text {Polymer solution }}=$ viscosity of polymer solution and $\eta_{\text {Solvent }}=$ viscosity of the solvent the polymer was dissolved in. An inflection point in the slope of this graph marks the onset of different regimes of polymer chain entanglement, namely, dilute, semidilute unentangled, and semidilute entangled regimes [3, 8, 23, 27-30].

2.7. Calculation of Dimensionless Parameters. Jet exit velocity $(U)$ determines how fast a polymer solution exits the reservoir orifice during centrifugal jet spinning. The jet exit velocity is defined as follows:

$$
U=\sqrt{\left(\mathrm{V}^{2}+\mathrm{R}^{2} * \omega^{2}\right)}
$$

where

$$
V=h * \frac{(D / 2)^{2}}{R^{2} * t},
$$

and $D=$ diameter of the orifice, $t=$ duration of each run, $h$ = height of the polymer solution within the reservoir, $R$ $=$ radius of reservoir, and $\omega=$ rotational speed in $\mathrm{rad} \cdot \mathrm{s}^{-1}[3]$.
The dimensionless parameters of interest in this study were the Capillary $(\mathrm{Ca})$ number:

$$
\mathrm{Ca}=\frac{\mathrm{We}}{\mathrm{Re}},
$$

which is in turn calculated using the Reynolds (Re) and Weber (We) numbers, given by

$$
\begin{gathered}
\operatorname{Re}=\frac{\rho U D}{\eta}, \\
\mathrm{We}=\frac{\rho U^{2} D}{\gamma} .
\end{gathered}
$$

where $\rho=$ density, $\eta=$ viscosity, and $\gamma=$ surface tension of the polymer solution. Ca represents the ratio of centrifugally induced shear viscous forces to surface tension forces and is thought to be a key predictor of polymer solution breakup and bead formation [3]. All these dimensionless numbers are reported in Table 1.

2.8. Statistical Analysis. All data are expressed as mean \pm standard error. A total of $n=4$ samples were tested for all nanofiber conditions. Statistical analysis was performed with one-way or two-way analysis of variance (ANOVA) for normally distributed data. The Pearson (for normally distributed data) and Spearman (for nonnormally distributed data) evaluations were used to analyze the correlation of the dimensionless numbers to morphological and mechanical properties of the fibers. A $p$ value of less than 0.05 was considered statistically significant.

\section{Results}

3.1. Characterization of Nanofibers Fabricated via Centrifugal Jet Spinning. All nanofiber conditions were fabricated in the small-scale CJS with the polymer jets being extruded out of the orifices due to the effect of hydrostatic and centrifugal forces exceeding the capillary forces, resulting in solvent evaporation and wrapping of nanofibers onto the surface of the reservoir (Figure 2(a)) [4, 19, 25]. Figure 3 shows a series of SEM images of the fabricated PCL fibers with increasing speeds and polymer concentrations. We observed the presence of beading at lower PCL concentrations and the absence of beads in fibers fabricated from higher PCL concentrations.

Fiber alignment for each distinct condition was found to be above 0.8 on a scale of $0-1.0$ with 0 being randomly oriented and 1.0 being perfectly aligned (Figure 4(a)) [3, $4,25,26]$. Both rotational speeds and polymer concentrations did not have a significant effect on fiber alignment $(p>0.05)$. Fiber diameter significantly $(p<0.05)$ decreased with increasing rotational speeds for each polymer composition (Figure 4(b)), and we observed a positive trend $(p=0.11)$ between nanofiber diameter and polymer concentration while keeping the rotational speed constant. It was also noted that the overall scaffold porosity (Figure 4(c)) was between 49 and 53\%, and it did not change with respect to speed or concentration. The SEM images were further 


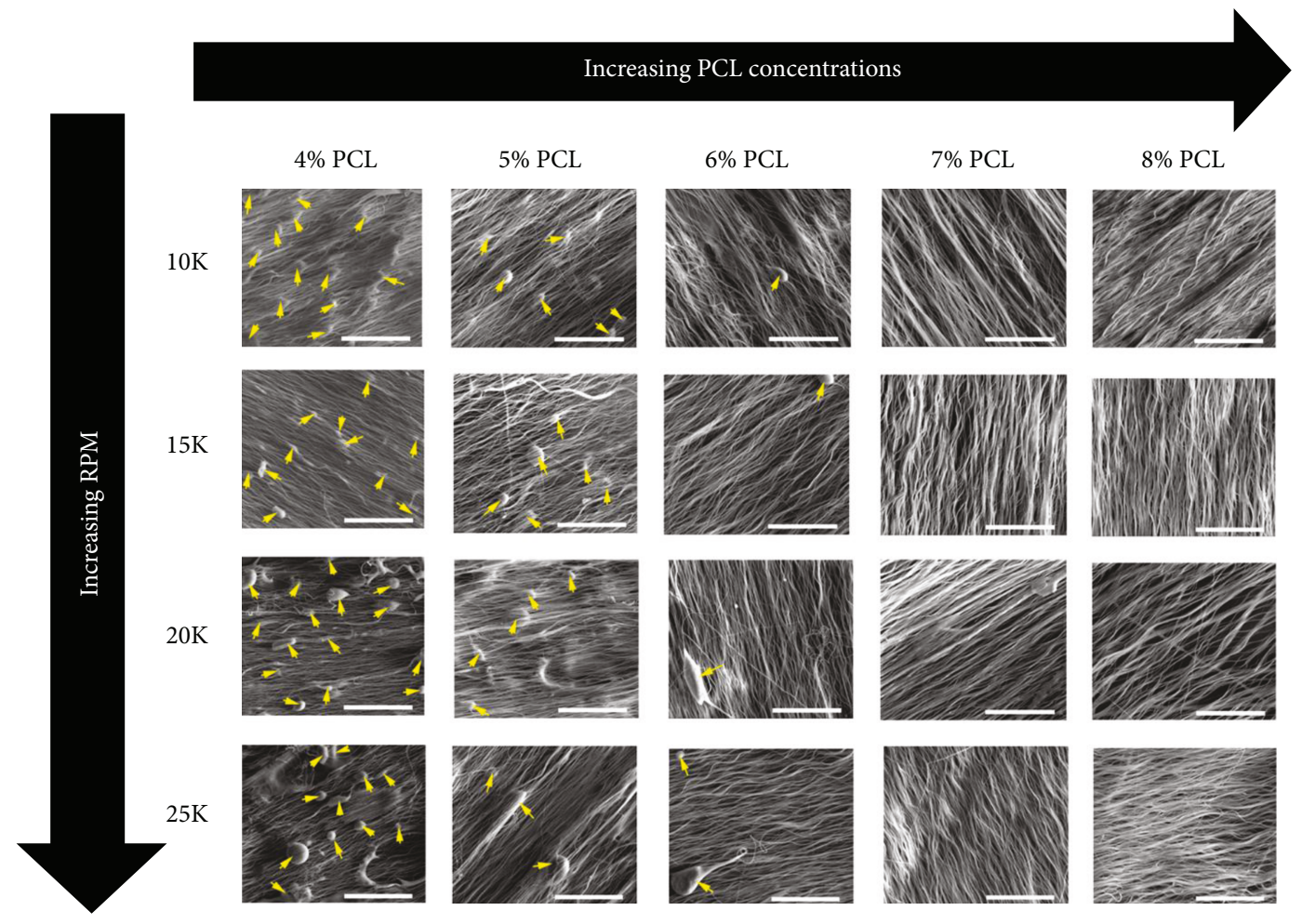

FIgURE 3: Representative scanning electron microscopy images of all PCL nanofiber conditions arranged with increasing speeds and concentrations. The yellow arrows mark the location of beads (scale bar $=50 \mu \mathrm{m}$ ).

analyzed for bead frequency, and it was observed that the average number of beads per area significantly $(p<0.05)$ decreased (Figure $4(d)$ ) with higher solution concentrations. The aspect ratios of beads were in the range of 2-4 and did not significantly differ between the polymer solutions and rotational speeds (Figure 4(e)).

\subsection{Calculating Critical Polymer Entanglement Concentration.} Polymer solution viscosity plays a crucial role in fiber morphology and determines the extent of polymer chain entanglement, which at high levels, promotes formation of continuous nanofibers $[14,23]$. Polymer chain entanglement refers to the interlocking of polymer chains during nanofiber fabrication as a result of chain overlapping [3, 23, 27]. Several studies have shown that at lower viscosities, the individual polymer chains are far apart resulting in beads or beaded fibers [23, 27, 29-33]. Within the analyzed polymer concentrations, distinct inflection points in the slope of the specific viscosity vs. concentration plots (Figure 5(a)) were observed which marked the different polymer chain entanglement regimes as outlined earlier. When the polymer solutions are dilute ( $C \ll C_{\text {critical }}$, dilute regime), the polymer chain entanglement that is necessary to form continuous fibers is not achieved because the distance between the polymer chains is relatively high, resulting in beads $[5,8,14]$. Under the semidilute unentangled regime $\left(C<C_{\text {critical }}\right)$, the distance between neighboring chains decreases but is still not significant enough and thus results in beaded fibers as shown for polymer concentrations between $4 \%<C<6 \%$. The individual polymer chains become sufficiently close enough under the semidilute entangled regime $\left(C>C_{\text {critical }}\right)$ to undergo internal entanglement, resulting in continuous fibers. These distinct regimes help determine the critical concentration (6\%) for the PCL solutions.

A phase diagram (Figure 5(b)) depicts the relationship between fiber morphology, capillary number, and polymer concentration at different rotational speeds. At lower concentrations and lower $\mathrm{Ca}$, fibers with beads were more commonly observed. From Figure 5(b), it can be noted that for fabricating continuous, bead-free PCL fibers, the polymer concentration should be higher than the critical concentration $(>6 \%)$ and should possess higher $\mathrm{Ca}(>25)$. Thus, finding the critical concentration of a polymer is crucial, as it may provide us information on gross nanofiber morphology. The angular velocity given by the $\mathrm{Ca}$ number must also be sufficiently high to overcome the surface tension resulting in stabilized polymer jets and reduced beading $[3,5]$.

3.3. Uniaxial Mechanical Testing. A representative stressstrain plot from our uniaxial tensile tests is shown in Figure 6(a). LM was determined using the slope of the linear region (between 5 and $20 \mathrm{MPa}$ ) and UTS was determined using the highest point on the curve $[4,19]$. We noted no significant difference in LM and UTS between the different rotational speeds (Figures 6(b) and 6(c)). However, there was a significant difference between nanofibers fabricated from 4 to $5 \%$ PCL concentrations compared to those from 6 to $8 \%$ PCL concentrations which had higher LM and UTS.

3.4. Analysis of Dimensionless Numbers and Validation with Scaled-Up CJS. From Table 1, we note that with an increase in polymer concentration, the dynamic viscosity $(\eta)$, surface 


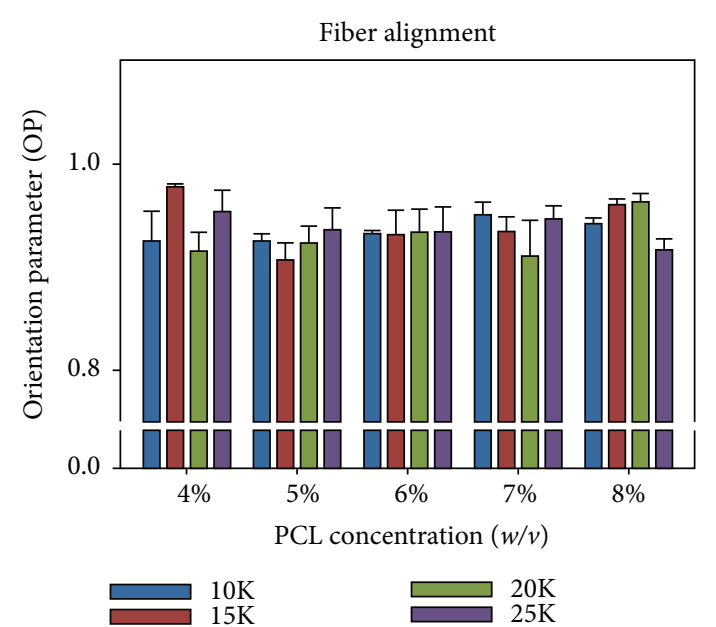

(a)

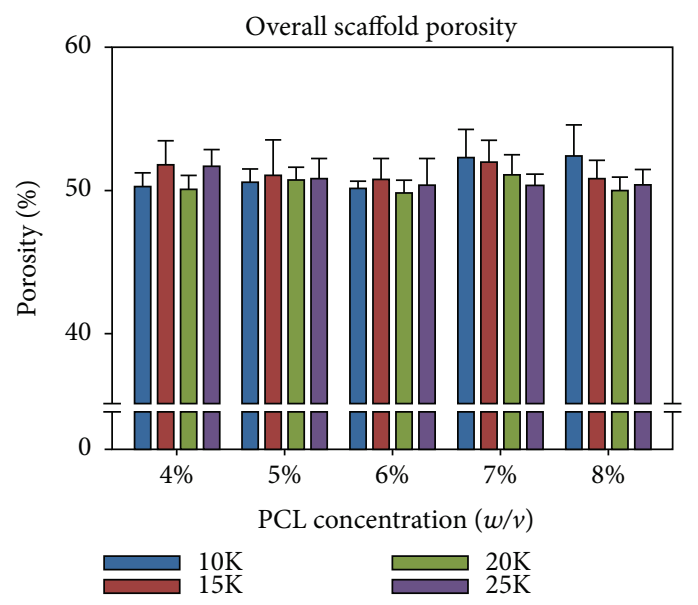

(c)

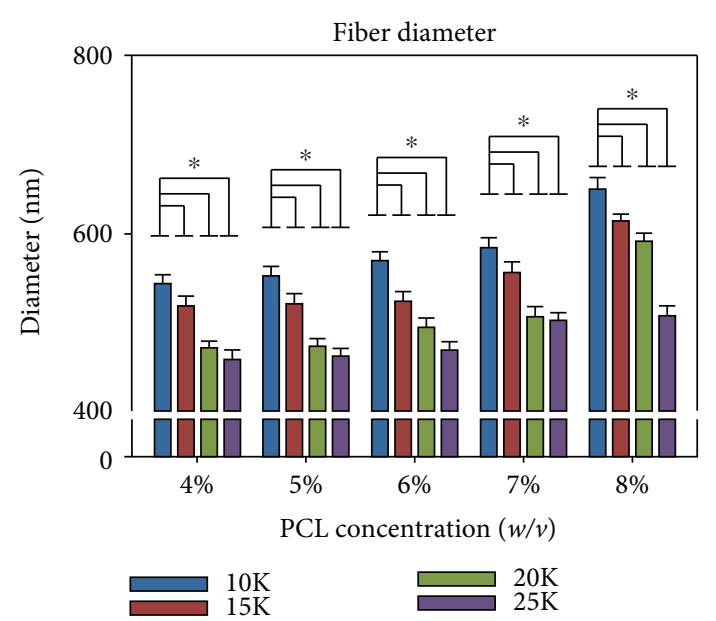

(b)

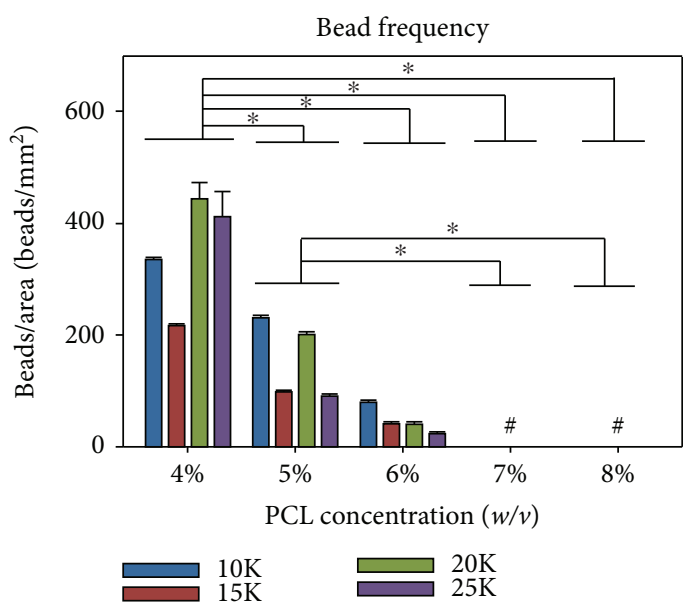

(d)

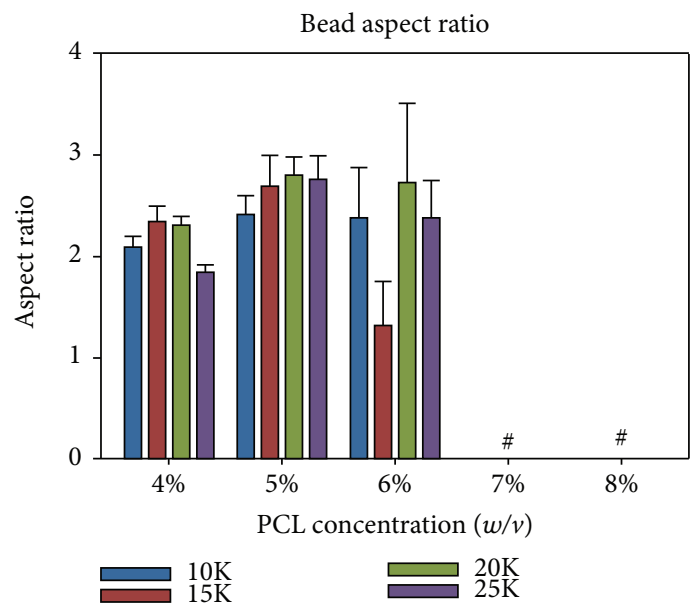

(e)

FIGURE 4: Morphological analyses of all PCL nanofiber conditions fabricated using the small-scale CJS prototype. (a) Fiber alignment, (b) fiber diameter, (c) scaffold porosity, (d) bead frequency, and (e) bead aspect ratio. 7-8\% PCL concentrations in subsets (d) and (e) are marked with \# to represent no beads. $(n=4$; $*$ denotes $p<0.05$. $)$

tension $(\gamma)$ and density $(\rho)$ all increased, with $\eta$ increasing by the largest magnitude. Correlation analysis was carried out by plotting the various dimensionless numbers with the fiber and bead morphology as well as mechanical properties to find which dimensionless number had the most significant effect in determining the fiber properties. Supplemental 


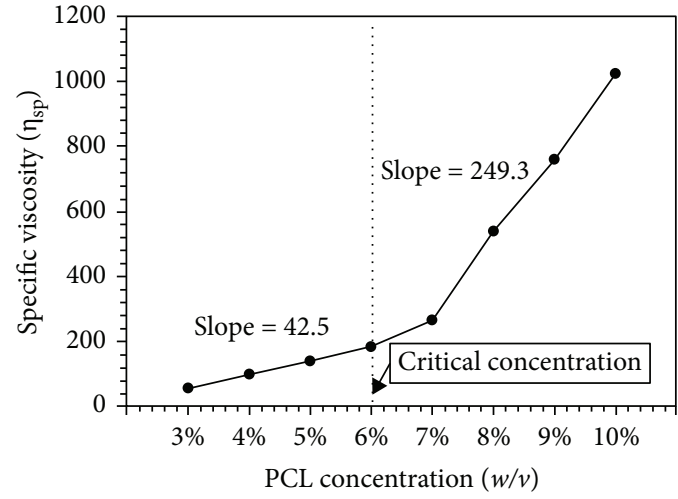

(a)

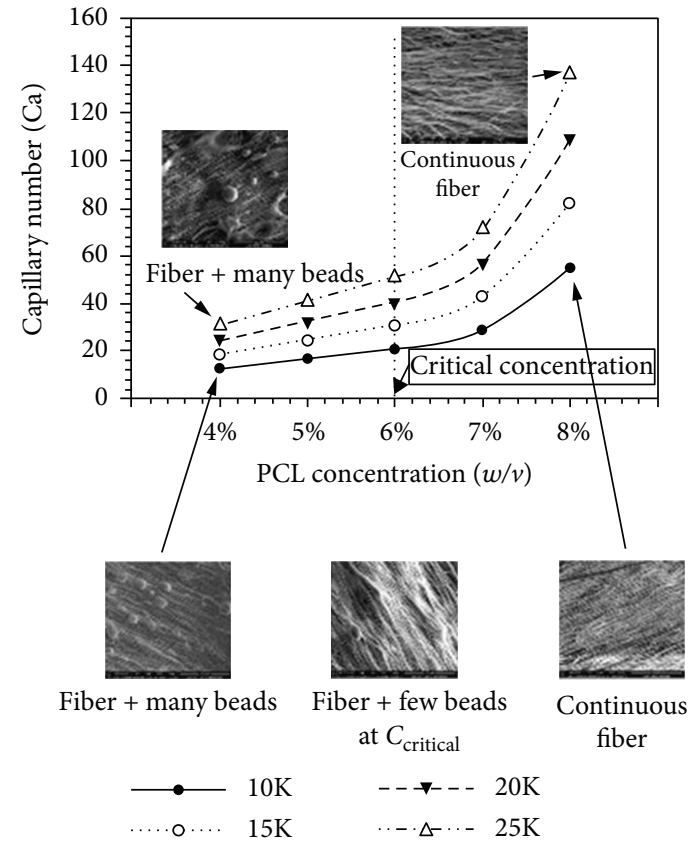

(b)

FIgURE 5: (a) Specific viscosity of PCL solutions plotted against the polymer concentration. The $C_{\text {critical }}$ was found to be 6 wt $\%$ PCL. (b) Phase diagram relating commonly observed fiber morphologies with Capillary number and PCL concentration. $(n=4$.)

Figures 1-3 show the correlation graphs for dimensionless numbers plotted against fiber alignment, fiber diameter, fiber porosity, bead frequency, bead aspect ratios, linear modulus, and ultimate tensile strength. We observed no significant correlation between fiber alignment, overall scaffold porosity, and the dimensionless numbers $(\mathrm{Ca}, \mathrm{Re}$, or $\mathrm{We})$ (Supp. Figure $1 \mathrm{~A}, \mathrm{C}$ ), but the $\mathrm{Re}$ and We numbers had significant negative correlations $(r=-0.831, p=0.0000057$ and $r=-0.758, p=0.0001$, respectively) with fiber diameter (Supp. Figure 1B). We also report (Supp. Figure 2A) a significant coefficient of correlation for $\mathrm{Re}$ and bead frequency $(r=0.575, p=0.05)$, where higher Re led to more bead occurrence. We also noted a trend that with higher $\mathrm{Ca}$ the bead frequency reduced $(r=-0.507, p=0.093)$. We observed that there was no significant correlation between the dimensionless numbers and bead aspect ratio (Supp. Figure 2B). Analyzing the correlation between dimensionless numbers and scaffold mechanical properties (Supp. Figure 3) revealed that there was a significant positive correlation of Ca with LM $(r=0.789, p=0.0000002)$ and UTS $(r=0.608, p$ $=0.0045)$. We also noticed that $\operatorname{Re}$ had a significant negative correlation with LM $(r=-0.618, p=0.0037)$ and UTS $(r=-0.594, p=0.0058)$.

As Re showed multiple significant correlations with fiber scaffold characteristics as depicted in Table 2, we matched this dimensionless parameter in the scaled-up CJS system and validated the ability of Re to predict fiber morphology with other polymers. Supplemental Table 1 reports the speeds that were calculated to match the Re numbers of the CJS prototype with that of the scaled-up CJS for the PCL polymer conditions. We found the critical polymer concentrations for all the polymer solutions and chose conditions that were higher than the $C_{\text {critical }}$ for each of the conditions. It was noted that the polymer concentration range tested for PVP-L solutions remained in the dilute regime and failed to reach $C_{\text {critical }}$. We calculated the appropriate rotational speeds required for the scaled-up CJS by matching the Re number of the prototype CJS. With this predetermined information, we spun fibers using 7\% PCL, 8\% PCL, 5\% PEO, 8\% PLA, 10\% PVP-L, and 5\% PVP-H solutions with the scaled-up CJS. We used PCL solutions to match the Re obtained from the small-scale CJS prototype operated at 10,000 rpm (7\% PCL-1 and 8\% PCL) and $15,000 \mathrm{rpm}$ (7\% PCL-2). All other polymer solutions were matched to the Re number of $8 \%$ PCL spun at $25,000 \mathrm{rpm}$ in the small-scale CJS prototype. Figure 7 shows the accuracy of matching the dimensionless numbers of newly fabricated nanofibers compared with predicted fiber diameter values obtained from the correlation data (Supp. Figure $1 \mathrm{~B}$ ). It was found that by matching the Re number, we were able to synthesize fibers with a prediction error ranging between 10 and 15\% for PCL and PVP-H. As expected, PVP-L resulted in only beads since the polymer solutions were dilute. The errors were larger when Re was matched to fabricate fibers from PEO $(\sim 18 \%)$ and PLA $(\sim 20 \%)$. We also noticed that the prediction error decreased among polymers dissolved in HFIP (Figure 7(a)). This may imply that the properties of the solvent play a role towards the resulting fiber properties and thus warrants further investigation. Considering dimensionless numbers that incorporates the solvent evaporation rate into account may help in reducing these prediction errors and build a more 

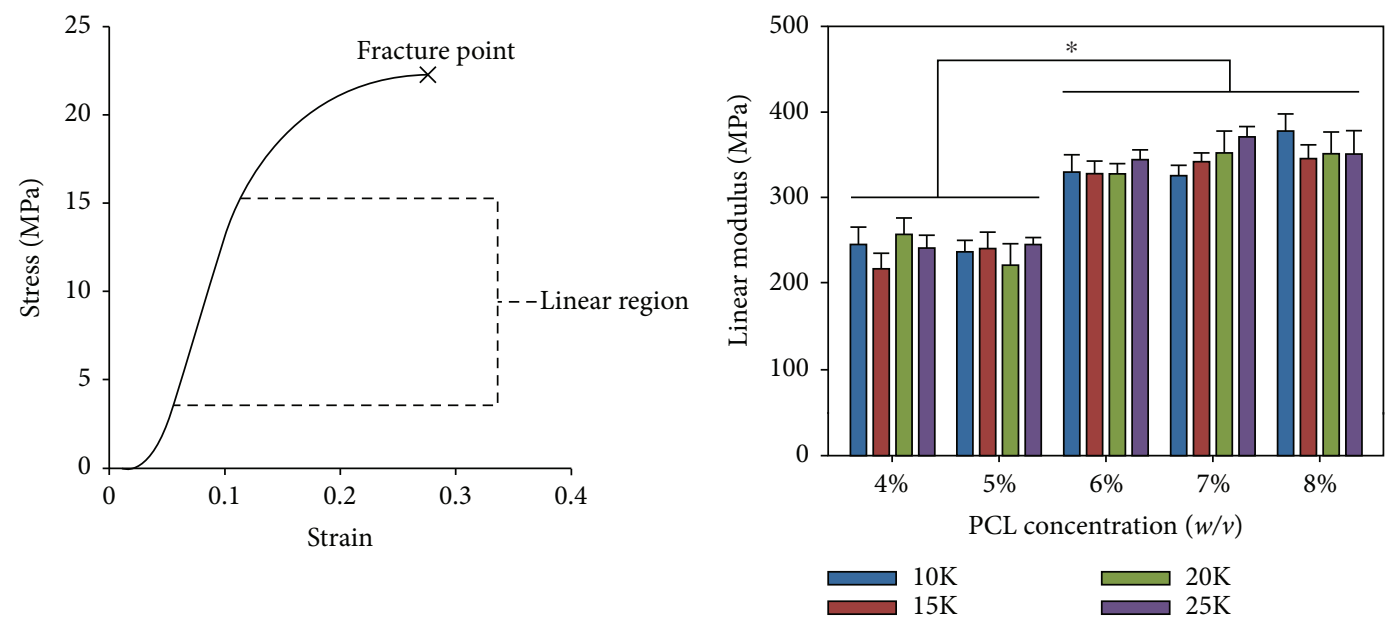

(a)

(b)

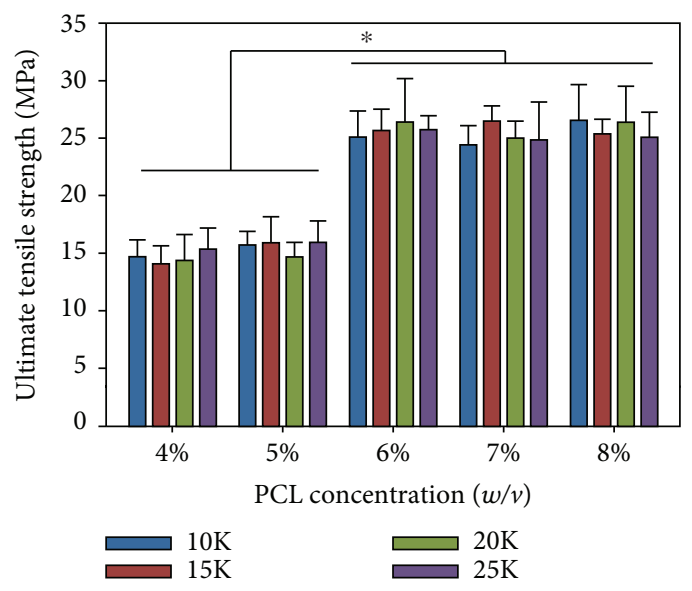

(c)

Figure 6: Mechanical characterization of PCL nanofibers. (a) Representative tensile stress-strain curve of 4\% PCL concentration spun at 10,000 rpm. (b) Measured linear modulus for PCL nanofiber conditions. (c) Measured ultimate tensile strength for PCL nanofiber conditions. $(n=3$; $*$ denotes $p<0.05$. $)$

TABLE 2: Summary of correlation analysis with $+/$ - denoting positive and negative significant correlation. NS denotes no significance.

\begin{tabular}{lccccccc}
\hline & $\begin{array}{c}\text { Fiber } \\
\text { alignment }\end{array}$ & $\begin{array}{c}\text { Fiber } \\
\text { diameter }\end{array}$ & $\begin{array}{c}\text { Scaffold } \\
\text { porosity }\end{array}$ & $\begin{array}{c}\text { Bead } \\
\text { frequency }\end{array}$ & $\begin{array}{c}\text { Bead aspect } \\
\text { ratio }\end{array}$ & $\begin{array}{c}\text { Linear } \\
\text { modulus }\end{array}$ & $\begin{array}{c}\text { Ultimate tensile } \\
\text { strength }\end{array}$ \\
\hline Capillary number & NS & NS & NS & NS & NS & + & NS \\
Weber number & NS & - & NS & NS & NS & NS & - \\
Reynolds number & NS & - & NS & + & NS \\
\hline
\end{tabular}

accurate model. All the fibers produced using the scaled-up CJS were highly aligned and their mechanical properties are summarized in Supplemental Table 2. These varied mechanical properties could be owed to several factors like fiber diameter, amorphous/crystallinity of the polymer, and fiber alignment $[5,34]$.

\section{Discussion}

Nanofiber scaffolds have the potential to be used for a wide range of applications, ranging from filtration to tissue scaffolding, underlining the importance of studying the large- scale production of these materials $[1,2,35]$. For the successful scale-up of any manufacturing process, knowledge of the key process parameters is crucial for repeatable and successful fabrication. Most mass production devices that produce polymeric fiber meshes have focused on optimizing electrospinning designs since this technique has been extensively studied and has long been understood [7, 36-41]. Some of the influencing parameters involved in predicting the electrospun fiber morphology can be used to understand the CJS process as it is relatively close to the behavior of polymer jets. However, the major drawbacks of these electrospinning methods are the high costs, low production rates, and electric 


\begin{tabular}{|c|c|c|c|c|c|c|c|}
\hline & & & \multicolumn{4}{|c|}{ Scaled-up CJS } \\
\hline & $\begin{array}{c}\text { Polymer } \\
\text { concentration }\end{array}$ & $\begin{array}{c}\text { Solvent } \\
\text { used } \\
\end{array}$ & $\begin{array}{c}\text { Reynolds } \\
\text { number } \\
(w / v) \%\end{array}$ & $\begin{array}{c}\text { Fiber } \\
\text { feature }\end{array}$ & $\begin{array}{c}\text { Predicted } \\
\text { fiber } \\
\text { diameter } \\
(\mathrm{nm})\end{array}$ & $\begin{array}{c}\text { New } \\
\text { fiber } \\
\text { diameter } \\
(\mathrm{nm})\end{array}$ & $\begin{array}{c}\text { Prediction } \\
\text { error } \\
(\%)\end{array}$ \\
\hline (i) & $7 \%$ PCL-1 & HFIP & 13.116 & Fiber & 572.69 & 661.24 & $13.39 \%$ \\
(ii) & $7 \%$ PCL-2 & HFIP & 19.674 & Fiber & 558.65 & 625.60 & $10.70 \%$ \\
(iii) & $8 \%$ PCL & HFIP & 6.584 & Fiber & 586.68 & 692.20 & $15.24 \%$ \\
(iv) & $5 \%$ PEO & Chloroform & 16.461 & Fiber & 565.53 & 692.68 & $18.36 \%$ \\
(v) & $8 \%$ PLA & Chloroform & 16.461 & Fiber & 565.53 & 707.11 & $20.02 \%$ \\
(vi) & $10 \%$ PVP-L & HFIP & 16.461 & Beads & 565.53 & N/A & N/A \\
(vii) & $5 \%$ PVP-H & HFIP & 16.461 & Fiber & 565.53 & 631.61 & $10.46 \%$ \\
\hline
\end{tabular}

(a)

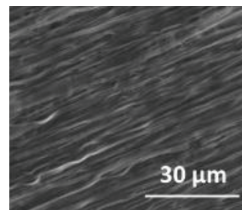

(i) $7 \% \mathrm{PCl}-1$

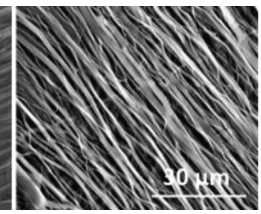

(ii) $7 \% \mathrm{PCl}-2$

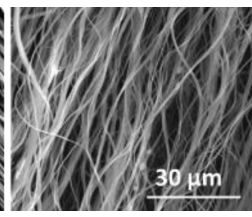

(iii) $8 \% \mathrm{PCl}$

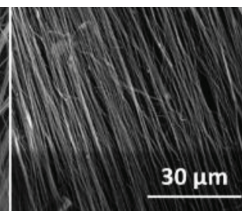

(iv) $5 \% \mathrm{PEO}$

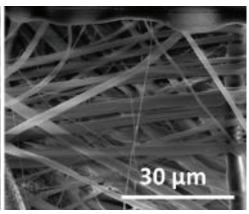

(v) $8 \%$ PLA

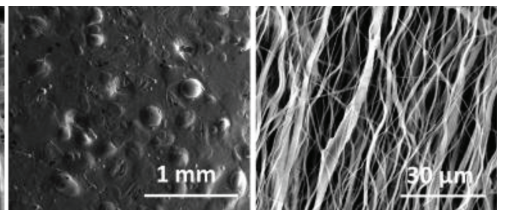

(vi) $10 \%$ PVP-L (vii) 5\% PVP-H

(b)

FIGURE 7: Validation study with all the different polymer-solvent conditions used to fabricate fibers by matching Re number in the scaled-up CJS. (a) Table comprising of all the polymer-solvent combinations, their fiber features, along with predicted and actual fiber diameters obtained from fibers fabricated using the scaled-up CJS $(n=4)$. (b) Representative SEM images for the different fiber meshes produced with the scaled-up CJS.

field interference that occurs when the number of jets are increased [7]. This has led researchers to study other nanofiber production techniques that use physical forces as opposed to electrostatic forces. CJS is one such emerging technique that can be industrially scaled-up for large-scale nanofiber fabrication at low costs $[9,10,14,25]$. Only a few studies exist that have investigated the factors involved in the CJS fabrication process experimentally [3, 8, 12-15, 17], with some focused on computational modelling [42-45]. However, these studies focused on fibers that were deposited on a collector placed away from the reservoir. In contrast, this study clearly highlights the utility of Re, We, and Ca to accurately determine a priori the fabrication parameters for a desired CJS nanofiber morphology with the fibers collected directly on the reservoir.

Our data suggests that we were able to fabricate fibers on the surface of the reservoir with high alignment, which have potential to be used as scaffolds for tissue engineering applications where there is a need to mimic tissues like muscle, blood vessels, or heart valves that have inherent alignment in their structure $[19,22,25,46]$. We also noted that the fiber diameter decreased with increasing speeds, which could be attributed to the result of increased extension and thinning of the polymer jet stream during the extrusion process $[3,4$, 25 ]. A trend of increased fiber diameter with increasing polymer concentration when the fibers were fabricated using the same rotational speed was observed, which could be a result of increased polymer content and solution viscosity $[8,13$, $14,23]$. We did not test any polymer concentrations below $4 \%(w / v)$ because our preliminary studies showed that these concentrations only produced beads. This is likely due to the Plateau-Rayleigh instability where the solution exiting the orifice forms droplets to minimize surface tension [10, $12,27,28]$. It can be noted that beading of fibers decreased with increasing polymer concentrations from our SEM images (Figure 3). Beaded fibers, as observed in lower concentrations, occur when the extended polymer jet undergoes solvent evaporation after the jet instability had set in. There is clearly a range beyond this which no beading was observed. This effect can also be owed to the role of chain entanglement for defining the various regions and that the critical concentration for continuous fiber formation was found to be $6 \%$ for PCL as depicted in Figure 5(a) $[8,27,29,32]$. As the polymer concentration increased along with an increased $\mathrm{Ca}$ number (Figure 5(b)), we were able to produce continuous nanofibers with no beads. This suggests that during the fiber extrusion process, higher rotational speeds cause the nanofibers to stretch to a greater extent, resulting in more efficient solvent evaporation and individual polymer chain entanglement that stabilized the polymer jet yielding continuous fibers. At lower speeds, the chain entanglement is not sufficient enough, leading to beaded fiber formation [3, 23, 27]. Furthermore, our mechanical data (Figure 6) shows that the LM and UTS are significantly higher for fibers fabricated from polymer concentrations that surpass the critical concentration. This suggests that the presence of beads in lower-concentration fibers (Figures 3, 4(d), and 4(e)) could have an effect on its mechanical properties.

We were able to produce fibers with overall scaffold porosities ranging between 49 and 53\%. Limited literature exists that has studied the porosity of CJS-spun fibers, and they show that CJS-spun fibers with similar porosities allow for cellular infiltration into the scaffold $[25,26,47]$. We speculate that the fibers collected on the reservoir at high rotational speeds undergo a rapid pull, resulting in the denser packing of the fibers and yielding lower porosity ranges. This can be overcome by introducing a collector placed at a distance from the reservoir. Alternatively, the addition of a 
protein component like gelatin to the polymer solution can increase fiber porosity [47]. It was reported that this addition yielded a higher porosity for PCL/gelatin blends compared to pure PCL fibers.

We performed correlation analysis to understand how the different fiber scaffold properties were affected by the dimensionless parameters, namely, $\mathrm{We}, \mathrm{Re}$, and $\mathrm{Ca}$ that are involved in the thinning of polymer jet filaments. We report all the significant and insignificant effects of dimensionless numbers on the fiber features to provide a thorough and holistic study. Our data suggests that there was a significant negative correlation between Re and We and fiber diameter. We know that Re and We are inversely proportional to viscosity and surface tension, respectively, and directly proportional to jet exit velocity. This suggests that viscous forces and surface tension of polymer solutions play a crucial role in determining fiber diameter during the fabrication process. The increased jet exit velocity may have resulted in decreased fiber diameter due to the longer extrusion of the polymer solution with increased centrifugal forces before solvent evaporation [13].

The use of computational models as a theoretical platform to understand the jet trajectory that occurs during the spinning process has been previously reported [42-45]. Divvela et al. studied the effects of polymer jet trajectory and diameter in the CJS process, and they reported similar findings where a decreasing Re resulted in the reduced thinning of the fibers [42]. This effect was attributed to the increase in viscous force that inhibits the extension of the polymer jet. They also observed steadier jet trajectories with a higherRenumber which can reduce the amount of fiber malformations. Contrary to our findings, the study reported that the We number did not affect the fiber diameter. A possible reason for this discrepancy could be due to solvent evaporation, which was not considered in the model by Divvela et al. In addition, their model did not account for the bending and twisting stiffness of the fibers which can occur during the CIS process. Recent work by Liu and Parker developed a more accurate and complex computational model that involved the bending and twisting stiffness of the fibers [43]. They showed that small fiber diameters and higher alignment can be attained by balancing the viscous and elastic forces. Their computational model demonstrated that when the polymer jet trajectory behavior is more elastic-like, the fiber jets will fall on the fast-rotating reservoir similar to our experimental observations. It is to be noted that this model did not include solvent evaporation and solidification of the fiber jets that can play a crucial role in determining the eventual trajectory and fabricated fiber morphology.

We observed that Re had a significant correlation with bead frequency. The reduction in beading can be attributed collectively to the increasing solution viscosity and spinning velocity leading to continuous fibers $[8,12-14,25,31]$. We matched the Re in the scaled-up CJS and carried out validation by fabricating fibers using other polymer-solvent combinations. We analyzed these newly fabricated fibers to compare their fiber characteristics. The fiber diameter data showed that the PCL and PVP-H fibers had comparatively lower prediction errors than PEO and PLA fibers
(Figure 7(a)). As shown in Figure 1, the solution evaporation rate plays an important role that can influence the fiber diameter. Since HFIP and chloroform have different solvent evaporation rates, this could have resulted in the changed prediction error. This is in agreement with other studies that investigated the effect of different solvents on fiber diameter $[8,12]$. These groups studied the role of elasticity and solvent evaporation rate on fiber diameter, but they did not consider the dimensionless numbers that were incorporated in our study. The representative SEM micrographs for the polymer solutions used for validation are provided in Figure 7 (b) (i-vii). We noticed that spinning PVP-L (Figure 7(b) (vi)) resulted in only beads since the concentration range tested was too dilute and fell short of the critical concentration. As stated earlier, fiber malformations can occur if the polymer concentrations are lower than their critical concentrations.

The utilization of other dimensionless numbers such as the Weissenberg number that investigates the elastic effects in response to the deformation rate of polymer solutions, the Rossby number that characterizes the effects of centrifugal and Coriolis forces, the Deborah number that studies the ratio of polymer relaxation time to flow, the elasticity number that investigates the role of elastic effects to inertial effects, and the processability number, which takes into account the role of solvent evaporation, might result in more accurate predictions for different polymer-solvent combinations $[5,8,12,13,42,43]$.

\section{Conclusions}

In conclusion, we demonstrate by using a small-scale prototype and a scaled-up CJS system that CJS-spun fiber morphologies can be predicted reasonably well a priori by a careful selection of dimensionless numbers, especially Re. We also demonstrate the accuracy of this method by validating with other polymer-solvent combinations like PEO-chloroform, PLA-chloroform, and PVP-HFIP. We show that by carefully studying the polymer solutions and matching the Re number, we can predict the fiber diameters with reasonable errors and are also able to create continuous and beadfree nanofibers. Overall, these findings can potentially help other researchers to design and construct similar CJS systems. In addition, this study provides valuable insight into the large-scale fabrication of fibers.

\section{Data Availability}

The data used to support the findings of this study are included within the article and the supplemental information.

\section{Disclosure}

We would like to acknowledge that a part of this manuscript was presented at the 8th Biennial Heart Valve Biology \& ?Tissue Engineering Meeting (London, United Kingdom) which was held on the 26th to the 28th of September, 2018, and at the 2018 Biomedical Engineering Society Annual Meeting 
(Atlanta, USA) which was held on the 17th to the 20th of October, 2018.

\section{Conflicts of Interest}

The authors declare that there is no conflict of interest regarding the publication of this paper.

\section{Authors' Contributions}

Prashanth Ravishankar and Alex Khang contributed equally to this work.

\section{Acknowledgments}

We acknowledge funding support from the Arkansas Biosciences Institute Grant 000167 and the State of Arkansas ?Student Undergraduate Research Fellowship (SURF). We would like to thank the University of Arkansas Institute for Nanoscience and Engineering for technical assistance with the scanning electron microscope. We would also like to thank Joshua A. Goss and Dr. Min Zou, Department of Mechanical Engineering, as well as Dr. Xianghong Qian, Department of Biomedical Engineering, University of Arkansas for assisting with the surface tension measurements. We also would like to acknowledge Ugochukwu Odega, Department of Biological Sciences, University of Arkansas, for his help with fabricating some of the fibers used in this study.

\section{Supplementary Materials}

Supplemental Figure 1: correlation analysis carried out for dimensionless numbers vs. (a) fiber alignment, (b) fiber diameter, and (c) scaffold porosity. Supplemental Figure 2: correlation analysis carried out for dimensionless numbers vs. (a) bead frequency and (b) bead aspect ratio. Supplemental Figure 3: correlation analysis carried out for dimensionless numbers vs. (a) linear modulus and (b) ultimate tensile strength. Supplemental Table 1: calculating the rotational speeds by matching the Re number from the small-scale CJS prototype to the new scaled-up CJS along with the dimensions of both the reservoirs employed. Supplemental Table 2: alignment and mechanical properties of fibers ?fabricated from a scaled-up CJS. (Supplementary Materials)

\section{References}

[1] R. L. Dahlin, F. K. Kasper, and A. G. Mikos, "Polymeric nanofibers in tissue engineering," Tissue Engineering Part B: Reviews, vol. 17, no. 5, pp. 349-364, 2011.

[2] R. Vasita and D. S. Katti, "Nanofibers and their applications in tissue engineering," International Journal of Nanomedicine, vol. 1, no. 1, pp. 15-30, 2006.

[3] M. R. Badrossamay, H. A. McIlwee, J. A. Goss, and K. K. Parker, "Nanofiber assembly by rotary jet-spinning," Nano Letters, vol. 10, no. 6, pp. 2257-2261, 2010.

[4] A. Khang, P. Ravishankar, A. Krishnaswamy et al., "Engineering anisotropic biphasic Janus-type polymer nanofiber scaffold networks via centrifugal jet spinning," Journal of Biomedical
Materials Research Part B: Applied Biomaterials, vol. 105, no. 8, pp. 2455-2464, 2017.

[5] J. J. Rogalski, C. W. M. Bastiaansen, and T. Peijs, "Rotary jet spinning review-a potential high yield future for polymer nanofibers," Nanocomposites, vol. 3, no. 4, pp. 97-121, 2017.

[6] F. A. A. Ruiter, C. Alexander, F. R. A. J. Rose, and J. I. Segal, "A design of experiments approach to identify the influencing parameters that determine poly-D, L-lactic acid (PDLLA) electrospun scaffold morphologies," Biomedical Materials, vol. 12, no. 5, article 055009, 2017.

[7] A. Valipouri, "Production scale up of nanofibers: a review," Journal of Textiles and Polymers, vol. 5, 2017.

[8] L. Ren, R. Ozisik, S. P. Kotha, and P. T. Underhill, "Highly efficient fabrication of polymer nanofiber assembly by centrifugal jet spinning: process and characterization," Macromolecules, vol. 48, no. 8, pp. 2593-2602, 2015.

[9] R. T. Weitz, L. Harnau, S. Rauschenbach, M. Burghard, and K. Kern, "Polymer nanofibers via nozzle-free centrifugal spinning," Nano Letters, vol. 8, no. 4, pp. 1187-1191, 2008.

[10] X. Zhang and Y. Lu, "Centrifugal spinning: an alternative approach to fabricate nanofibers at high speed and low cost," Polymer Reviews, vol. 54, no. 4, pp. 677-701, 2014.

[11] K. Sarkar, C. Gomez, S. Zambrano et al., "Electrospinning to Forcespinning ${ }^{\mathrm{TM}}$," Materials Today, vol. 13, no. 11, pp. 12-14, 2010.

[12] H. M. Golecki, H. Yuan, C. Glavin et al., "Effect of solvent evaporation on fiber morphology in rotary jet spinning," Langmuir, vol. 30, no. 44, pp. 13369-13374, 2014.

[13] P. Mellado, H. A. McIlwee, M. R. Badrossamay, J. A. Goss, L. Mahadevan, and K. Kit Parker, "A simple model for nanofiber formation by rotary jet-spinning," Applied Physics Letters, vol. 99, no. 20, article 203107, 2011.

[14] Y. Lu, Y. Li, S. Zhang et al., "Parameter study and characterization for polyacrylonitrile nanofibers fabricated via centrifugal spinning process," European Polymer Journal, vol. 49, no. 12, pp. 3834-3845, 2013.

[15] S. Padron, A. Fuentes, D. Caruntu, and K. Lozano, "Experimental study of nanofiber production through forcespinning," Journal of Applied Physics, vol. 113, no. 2, article 024318, 2013.

[16] L. Ren, R. Ozisik, and S. P. Kotha, "Rapid and efficient fabrication of multilevel structured silica micro-/nanofibers by centrifugal jet spinning," Journal of Colloid and Interface Science, vol. 425, pp. 136-142, 2014.

[17] Z. McEachin and K. Lozano, "Production and characterization of polycaprolactone nanofibers via forcespinning ${ }^{\mathrm{TM}}$ technology," Journal of Applied Polymer Science, vol. 126, no. 2, pp. 473-479, 2012.

[18] E. Chong, T. Phan, I. Lim et al., "Evaluation of electrospun PCL/gelatin nanofibrous scaffold for wound healing and layered dermal reconstitution," Acta Biomaterialia, vol. 3, no. 3, pp. 321-330, 2007.

[19] A. M. Loordhuswamy, V. R. Krishnaswamy, P. S. Korrapati, S. Thinakaran, and G. D. V. Rengaswami, "Fabrication of highly aligned fibrous scaffolds for tissue regeneration by centrifugal spinning technology," Materials Science and Engineering: $C$, vol. 42, pp. 799-807, 2014.

[20] N. L. Leong, N. Kabir, A. Arshi et al., "Evaluation of polycaprolactone scaffold with basic fibroblast growth factor and fibroblasts in an athymic rat model for anterior cruciate ligament reconstruction," Tissue Engineering Part A, vol. 21, no. 1112, pp. 1859-1868, 2015. 
[21] F. A. Petrigliano, G. A. Arom, A. N. Nazemi, M. G. Yeranosian, B. M. Wu, and D. R. McAllister, "In vivo evaluation of electrospun polycaprolactone graft for anterior cruciate ligament engineering," Tissue Engineering Part A, vol. 21, no. 7-8, pp. 1228-1236, 2015.

[22] V. A. Kumar, L. P. Brewster, J. M. Caves, and E. L. Chaikof, "Tissue engineering of blood vessels: functional requirements, progress, and future challenges," Cardiovascular Engineering and Technology, vol. 2, no. 3, pp. 137-148, 2011.

[23] L. Ren, V. Pandit, J. Elkin, T. Denman, J. A. Cooper, and S. P. Kotha, "Large-scale and highly efficient synthesis of microand nano-fibers with controlled fiber morphology by centrifugal jet spinning for tissue regeneration," Nanoscale, vol. 5, no. 6, pp. 2337-2345, 2013.

[24] G. H. McKinley, Dimensionless Groups for Understanding Free Surface Flows of Complex Fluids, pp. 6-9, Society of Rheology Bulletin, 2005.

[25] M. R. Badrossamay, K. Balachandran, A. K. Capulli et al., "Engineering hybrid polymer-protein super-aligned nanofibers via rotary jet spinning," Biomaterials, vol. 35, no. 10, pp. 3188-3197, 2014.

[26] A. K. Capulli, M. Y. Emmert, F. S. Pasqualini et al., "JetValve: rapid manufacturing of biohybrid scaffolds for biomimetic heart valve replacement," Biomaterials, vol. 133, pp. 229-241, 2017.

[27] S. L. Shenoy, W. D. Bates, H. L. Frisch, and G. E. Wnek, "Role of chain entanglements on fiber formation during electrospinning of polymer solutions: good solvent, non-specific polymerpolymer interaction limit," Polymer, vol. 46, no. 10, pp. 33723384, 2005.

[28] C. Wang, H. S. Chien, K. W. Yan et al., "Correlation between processing parameters and microstructure of electrospun poly(D,L-lactic acid) nanofibers," Polymer, vol. 50, no. 25, pp. 6100-6110, 2009.

[29] R. Rošic, J. Pelipenko, P. Kocbek, S. Baumgartner, M. BešterRogač, and J. Kristl, "The role of rheology of polymer solutions in predicting nanofiber formation by electrospinning," European Polymer Journal, vol. 48, no. 8, pp. 1374-1384, 2012.

[30] A. A. Vetcher, R. Gearheart, and V. N. Morozov, "Correlation of morphology of electrospun fibers with rheology of linear polyacrylamide solution," Polymer Journal, vol. 39, no. 8, pp. 878-881, 2007.

[31] C. C. Call and C. J. Schwartz, "The use of weber number to predict morphology in the electrospinning of poly(ethylene oxide) nanofibers," Journal of Applied Polymer Science, vol. 120, no. 2, pp. 880-885, 2011.

[32] L. J. Fetters, D. J. Lohse, and R. H. Colby, "Chain dimensions and entanglement spacings," in Physical Properties of Polymers Handbook, pp. 447-454, Springer, 2007.

[33] R. H. Colby, L. J. Fetters, W. G. Funk, and W. W. Graessley, "Effects of concentration and thermodynamic interaction on the viscoelastic properties of polymer-solutions," Macromolecules, vol. 24, no. 13, pp. 3873-3882, 1991.

[34] J. Bao, L. I. Clarke, and R. E. Gorga, "Effect of constrained annealing on the mechanical properties of electrospun poly(ethylene oxide) webs containing multiwalled carbon nanotubes," Journal of Polymer Science Part B: Polymer Physics, vol. 54, no. 8, pp. 787-796, 2016.
[35] K. Garg and G. L. Bowlin, "Electrospinning jets and nanofibrous structures," Biomicrofluidics, vol. 5, no. 1, article 013403, 2011.

[36] S. Nakano and K. Miyazawa, "Melt electrospinning technology for production of nanofibers-recent topics on spinning machines and their scale-up," Sen'i Gakkaishi, vol. 67, no. 9, pp. 259-265, 2011.

[37] H. Niu and T. Lin, "Fiber generators in needleless electrospinning," Journal of Nanomaterials, vol. 2012, Article ID 725950, 13 pages, 2012

[38] N. M. Thoppey, M. Roman, Q. Wang et al., "Unconfined fluid, needleless electrospinning for scale-up production of high quality nanofibers," in Abstracts of Papers of the American Chemical Society, vol. 247, American Chemical Society, 2014.

[39] L. Wang, M. W. Chang, Z. Ahmad, H. Zheng, and J. S. Li, "Mass and controlled fabrication of aligned PVP fibers for matrix type antibiotic drug delivery systems," Chemical Engineering Journal, vol. 307, pp. 661-669, 2017.

[40] L. Wang, C. Zhang, F. Gao, and G. Pan, "Needleless electrospinning for scaled-up production of ultrafine chitosan hybrid nanofibers used for air filtration," RSC Advances, vol. 6, no. 107, pp. 105988-105995, 2016.

[41] X. Wang, T. Lin, and X. Wang, "Scaling up the production rate of nanofibers by needleless electrospinning from multiple ring," Fibers and Polymers, vol. 15, no. 5, pp. 961-965, 2014.

[42] M. J. Divvela, A. C. Ruo, Y. Zhmayev, and Y. L. Joo, "Discretized modeling for centrifugal spinning of viscoelastic liquids," Journal of Non-Newtonian Fluid Mechanics, vol. 247, pp. 6277, 2017.

[43] Q. Liu and K. K. Parker, “A viscoelastic beam theory of polymer jets with application to rotary jet spinning," Extreme Mechanics Letters, vol. 25, pp. 37-44, 2018.

[44] S. Noroozi, H. Alamdari, W. Arne, R. G. Larson, and S. M. Taghavi, "Regularized string model for nanofibre formation in centrifugal spinning methods," Journal of Fluid Mechanics, vol. 822, pp. 202-234, 2017.

[45] S. M. Taghavi and R. G. Larson, "Regularized thin-fiber model for nanofiber formation by centrifugal spinning," Physical Review E, vol. 89, no. 2, article 023011, 2014.

[46] S. L. Liu, Y. Z. Long, Z. H. Zhang et al., "Assembly of oriented ultrafine polymer fibers by centrifugal electrospinning," Journal of Nanomaterials, vol. 2013, Article ID 713275, 9 pages, 2013.

[47] L. N. Zamproni, M. A. V. M. Grinet, M. T. V. V. Mundim et al., "Rotary jet-spun porous microfibers as scaffolds for stem cells delivery to central nervous system injury," Nanomedicine: Nanotechnology, Biology and Medicine, vol. 15, no. 1, pp. 98107, 2019. 


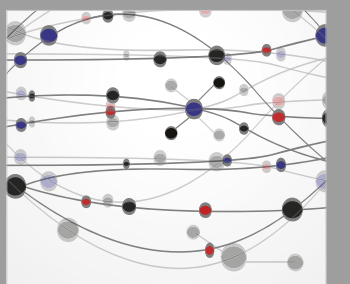

The Scientific World Journal
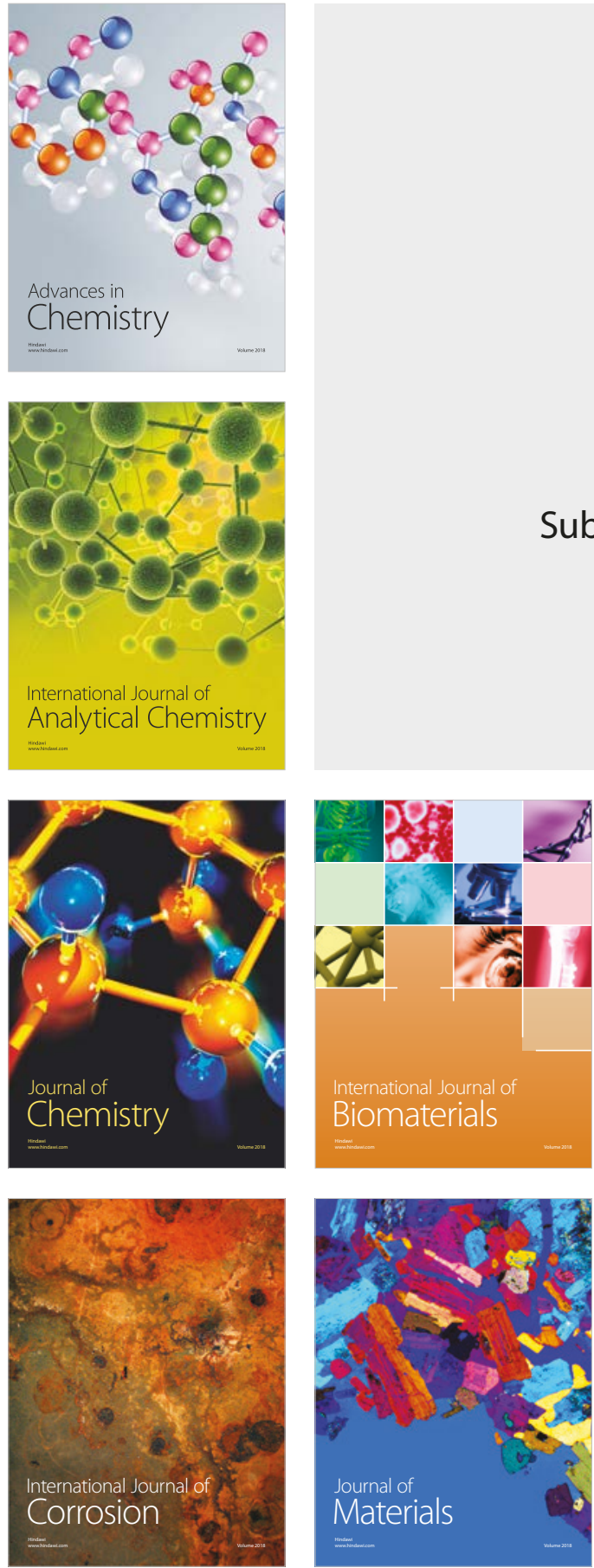

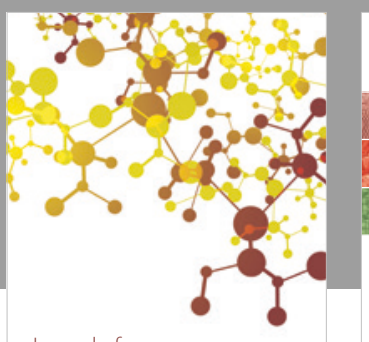

Journal of

Applied Chemistry
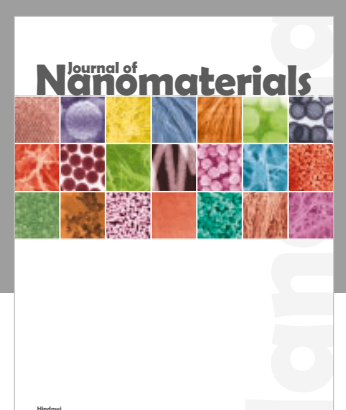

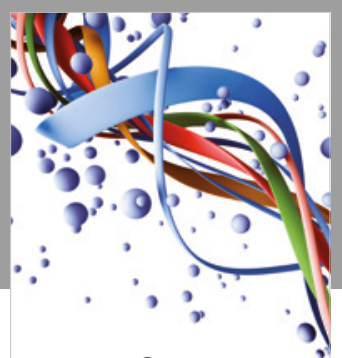

Scientifica

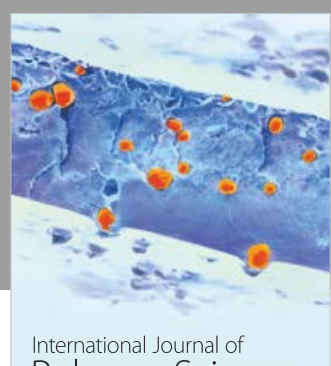

Polymer Science

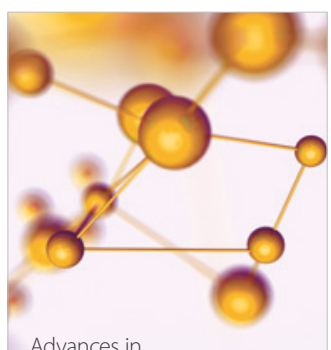

Physical Chemistry
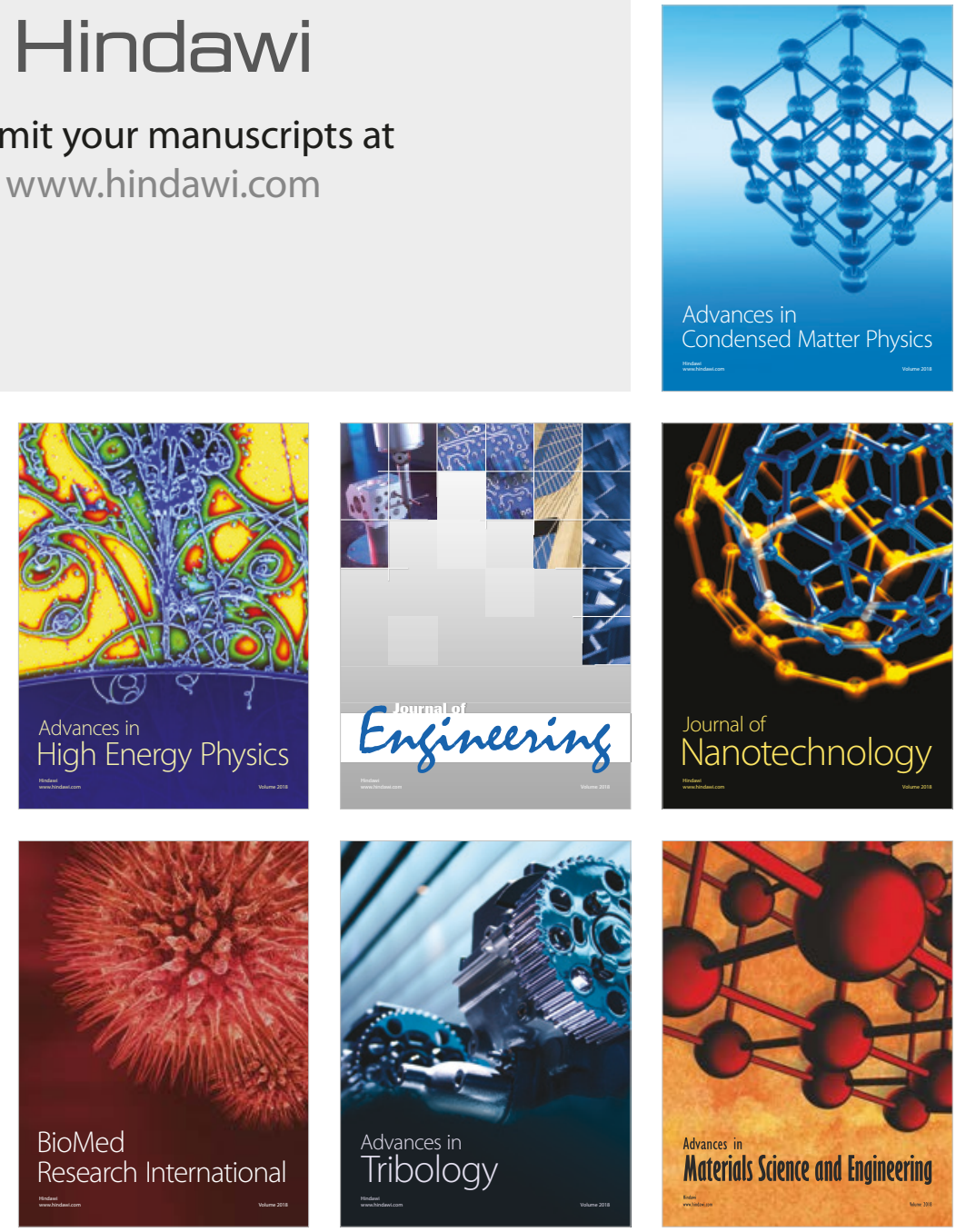